\title{
Perfil de nascimentos no Município do Rio de J aneiro: uma análise espacial
}

\author{
Birth profile for the city of Rio de J aneiro: \\ a spatial analysis
}

Eleonora d'Orsi 1

Marília Sá Carvalho 1

\footnotetext{
1 Departamento de Epidemi ol ogia e Métodos Quantitativos em Saúde, Escola Nacional de Saúde Pública, Fundação Oswaldo Cruz. Rua Leopoldo Bulhões 1480, Rio de Janeiro, RJ 21041-210, Brasil.
}

Abstract This articleanalyzes the birth profile by neighborhood in the city of Rio deJaneiro, using data for 1994 from the Data Base on Live Births. Pattern maps were employed in addition to the Moran I statistical test to detect spatial clustering. Proportions of live newborns with an Apgar score of 8-10, cesareans, mothers with greater than a secondary school education, and teenage mothers displayed visually identifiable spatial patterns and significant spatial self-correlation. Low birth weight displayed a random pattern, indicating that on this scale of analysis, this indicator does not distinguish risk groups, despite its unquestionable predictive value for child morbidity/mortality at the individual level. The Apgar score, despite the high number of non-responses in some neighborhoods, showed a pattern more consistent with the distribution of the neighborhoods and should thus be used more extensively. The methodology expanded the available knowledge on the birth profile in the city, showing potential for orienting measures devoted to specific geographic areas.

Key words Spatial Analysis; Birth; Birth Weight; Child Morbidity; Child Mortality

Resumo Neste artigo analisou-se o perfil de nascimentos por bairros do Município do Rio de Janeiro, utilizando-se os dados do Si stema de Informações sobre Nasci dos Vivos de 1994. Foram empregados mapas de padrão e o teste estatístico I de Moran para detecção de cluster espacial . As proporções de nasci dos vi vos com Apgar entre oito e dez, de cesáreas, de mães com escolaridade aci ma de segun do grau e de mães adol escentes apresentaram padrão espacial visualmente identificável e autocorrel ação espacial significativa. O baixo peso apresentou padrão espacial al eatório, demonstrando que, nesta escala de análise, este indicador não discrimina grupos de risco, apesar do seu inquestionável valor preditivo para morbi-mortalidade infantil em nível individual. O Apgar, apesar do el evado número de não-resposta em alguns bairros, apresenta padrão mais consistente com a distribui ção dos bairros, devendo, por isso, ser mais utilizado. Esta metodologia permitiu aprofundar o conhecimento sobre o perfil de nascimentos no Município, apresentando potencial no di recionamento de ações voltadas para áreas específicas.

Palavras-chave Análise Espacial; Nascimento; Peso ao Nascer; Morbidade Infantil; Mortalidade Infantil 


\section{Introdução}

Neste trabalho é analisado o perfil de nascimentos no Município do Rio de Janeiro, buscando-se identificar espacialmente áreas com características específicas, a fim de subsidiar programas ou políticas voltados para melhoria da saúde materno-infantil visando aumentar a eficiência na utilização de recursos públicos, foi utilizado um enfoque de risco usando informações que permitam a definição de áreas prioritárias de atuação (Hayes, 1992).

Foram utilizados dados secundários provenientes do Sistema de Informações sobre Nascidos Vivos (Sinasc) de 1994 para o Município do Rio de Janeiro, agregados por bairros. Esta divisão por bairros é fruto da forma de ocupação do espaço da cidade que foi, historicamente, condicionada por dois tipos de meios de transporte: os bondes em direção à Zona Sul, onde se localizaram as classes altas, e os trens em direção aos subúrbios da Zona Norte, ocupados pela população operária e de baixa renda. As reformas realizadas no início do século XX, com o objetivo de 'sanear'a cidade, destruíram os cortiços localizados em sua área central, acelerando o processo de favelização dos morros. A conformação urbana atual do Rio de Janeiro se assemel ha a um mosaico de áreas pobres e ricas intercaladas, fruto de um processo seletivo de aplicação de recursos públicos em infra-estrutura urbana que privilegiou os bairros da Zona Sul e al guns bairros da Zona Norte, de maior poder aquisitivo, em detrimento dos subúrbios das Zonas Norte e Oeste (Abreu, 1987). Cada bairro não constitui uma área homogênea, princi palmente devido à presença de favelas, havendo, inclusive, alguns bairros quase totalmente constituídos pelas mesmas.

O Sinasc é um subsistema de informações de âmbito nacional, sob responsabilidade das Secretarias Estaduais e Municipais de Saúde, concebido para diminuir a subenumeração e melhorar a qualidade da informação sobre nascidos vivos. Este sistema é alimentado pelas declarações de nascido vivo (DN), documento oficial emitido pelo hospital onde ocorre o nascimento. Avaliação do Sinasc no Estado de São Paulo demonstrou uma excel ente cobertura $(99,5 \%)$ e boa fidedignidade para quase todas as variáveis, exceto o índice de Apgar e o nível de instrução da mãe (Mello Jorge et al., 1993).

Entre as principais características epidemiológicas do recém-nascido, da gravidez e do parto incluídas na DN estão: índice de Apgar, peso ao nascer, duração da gestação e tipo de parto. O Apgar é um índice composto que mede a vitalidade do recém-nascido no primeiro e no quinto minuto após o nascimento; constitui-se por: cor da pele, respiração, batimentos cardíacos, tônus muscular e resposta a estímulos nervosos. Classificação proposta em 1981 por Segre (Mello Jorge et al., 1993) subdivide o Apgar em: boas condições ao nascimento (Apgar entre oito e dez), anóxia leve (Apgar igual a sete), anóxia moderada (Apgar entre quatro e seis) e anóxia grave (Apgar entre zero etrês).

O baixo peso e a prematuridade constituem-se os principais determinantes da mortalidade perinatal, estando também associados com importante parcela de morbidade infantil, incluindo comprometimento neuromotor, problemas respiratórios crônicos e infecções (Berkowitz \& Papiernik, 1993; Wilcox \& Skjfoerven, 1992; Gray et al., 1991). A atenção das pesquisas e programas de saúde tem se voltado para os bebês com baixo peso ao nascer (peso inferior a $2.500 \mathrm{~g}$ ) e com peso deficiente (entre 2.500 e $2.999 \mathrm{~g}$ ), que representam grupos vulneráveis ao impacto de condições ambientais e sociais.

Os nascimentos de baixo peso podem ser classificados em dois grupos fisiopatologicamente distintos: os prematuros e os bebês a termo com retardo de crescimento intra-uterino (RCIU). O segundo grupo está etiologicamente relacionado a fatores intrínsecos, como capacidade diminuída de transporte de nutrientes em nível placentário, fatores extrínsecos, como deficiências nutricionais antes e/ ou durante a gravidez, ou uma combinação de ambos. Alguns autores relacionam a alta incidência de baixo peso ao nascer encontrada principalmente em países em desenvolvimento a bebês com RCIU, enquanto a prematuridade seria o principal componente nos países desenvolvidos, em especial entre aqueles com as menores incidências de baixo peso. Dentre os fatores de risco associados com a ocorrência de bebês com RCIU estão incluídos: baixo nível materno de instrução, pré-natal inadequado, estado nutricional deficiente antes da gravidez, ganho de peso insuficiente durante a gestação, infecções geniturinárias, tabagismo, ausência de cônjuge e primi paridade (Kallan, 1993; Sanjose \& Roman, 1991; Puffer, 1987; Mavalankar et al., 1992; Ferraz et al., 1990). A prematuridade tem sido mais freqüentemente associada a fatores biológicos maternos, e o RCIU, a fatores sócio-econômicos, não havendo, entretanto, uma separação distinta, uma vez que fatores sócio-econômicos podem ser mediados por fatores biológicos maternos e cuidados pré-natais, indicando que a adequa- 
da atenção ao pré-natal e ao parto possibilita a diminuição do risco de baixo peso e prematuridade.

O tipo de parto permite caracterizar a prática obstétrica nos hospitais. O Brasil ocupa o primeiro lugar mundial na realização de cesáreas, com 36\% de partos operatórios em 1996 (PNAD, 1996), alcançando 48,4\% no Estado de São Paulo (Mello Jorge et al. 1993). Nos países europeus, os valores mais elevados situam-se em torno de $12 \%$ (Thiery \& Deron, 1986) e nos Estados Unidos houve um pico de $24,7 \%$ em 1988, decaindo para 22,8\% em 1993 (Porreco \& Thorp, 1996), originando vasta literatura a respeito. Entre os fatores que influenciam a opção por um parto operatório, têm sido relatados: a organização da atenção obstétrica, pautada pela conveniência de uma intervenção programada; treinamento insuficiente durante a formação médica para acompanhamento do parto vaginal; fatores institucionais ligados à forma de pagamento e exclusão do pagamento de anestesia peridural para partos vaginais pelo SUS; a questão da esterilização cirúrgica, realizada freqüentemente durante uma cesárea eletiva; fatores sócio-culturais, tais como medo da dor no parto normal e da perda da elasticidade vaginal, que levariam parte das mulheres a preferirem um parto cirúrgico (Faúndes \& Cecatti, 1991; Rattner, 1996). Esta situação evidencia uma importante distorção no modelo de atenção ao parto, submetendo mães e crianças a riscos desnecessários.

Em relação às características da mãe, estão presentes na DN a idade e o nível de instrução maternos. A idade materna é um importante fator de risco para baixo peso ao nascer e para mortalidade infantil, particularmente entre as adolescentes com menos de vinte anos e entre as mães com 35 anos e mais. O grau de instrução materno, única variável da DN que permite avaliar a situação sócio-econômica do recém-nascido, está associado com o resultado da gestação e sobrevivência no primeiro ano de vida (Mello Jorge et al., 1992).

Neste trabalho buscou-se detectar a existência de padrão espacialmente condicionado das variáveis selecionadas. A presença de autocorrelação espacial significa que áreas próximas apresentam taxas mais semelhantes do que seria esperado caso a distribuição fosse aleatória, indicando que o fenômeno estudado apresenta dependência espacial.

\section{Metodologia}

Foram utilizados os dados do Sinasc para 1994, geocodificados com o auxílio do campo referente ao bairro de residência da mãe, presente na DN. Em cada bairro foram calculadas, em relação ao total de nascidos vivos, as proporções de nascidos vivos com: Apgar bom, baixo peso, peso deficiente, peso favorável, prematuros, cesáreas, mães adolescentes, mães com idade igual ou superior a 35 anos e mães com escolaridade acima de segundo grau.

A análise estatística foi realizada utilizando-se os programas estatísticos SAS, S-PLUS e STAT!. As variáveis do Sinasc foram inicialmente submetidas a análise univariada e bivariada para o Município como um todo. Os indicadores agregados por bairro de residência da mãe foram submetidos novamente a análise univariada e construídos os boxpl ots das proporções por bairros e os mapas de padrão. Nestes, utilizaram-se como pontos de corte os quintis da distribuição de cada um dos indicadores por bairro. A fim de diminuir a flutuação aleatória, foram eliminados da análise aqueles bairros cujo número de nascimentos não atingisse o mínimo para garantir a estabilidade do indicador com 95\% de confiança. Utilizou-se a seguinte fórmula de cálculo de amostra:

$$
\begin{aligned}
& \mathrm{n}=\frac{\mathrm{z}^{2} \mathrm{pq}}{\mathrm{d}^{2}}, \text { sendo: } \\
& \mathrm{n}=\text { número mínimo de nascimentos; } \\
& \mathrm{z}=\text { valor na tabela } \mathrm{z} \text { para } 95 \% \text { de confiança }
\end{aligned}
$$
$(1,96)$;

$$
\mathrm{p}=\text { proporção estimada a partir da média }
$$
no Município;

$q=1-p ;$

d =amplitude estimada do intervalo contendo o indicador.

A medida de autocorrelação espacial, estatística I de Moran, é um coeficiente de autocorrelação ponderado, utilizado para determinar se áreas próximas são mais similares do que seria esperado por uma distribuição al eatória (Cliff \& Ord, 1981). Ele apresenta a forma clássica de qualquer coeficiente de autocorrelação: no numerador uma medida de covariância e no denominador uma medida de variância.

$$
\mathrm{I}=\frac{\mathrm{N} \sum_{i=1}^{N} \sum_{j=1}^{N} \mathrm{~W}_{i j} z_{i} z_{j}}{\mathrm{~S}_{0} \sum_{i=1}^{N} \mathrm{z}_{i}^{2}} \text {, sendo: }
$$

$\mathrm{N}=$ no de áreas, por exemplo (bairros);

$z_{i}=$ diferença entre indicador na área i e média geral $z_{i}=x_{i}-x$;

$w_{i j}=$ matriz de conexão entre as áreas i e j, onde $\mathrm{w}_{\mathrm{ij}}=1$, se i e j eram vizinhos e $\mathrm{w}_{\mathrm{ij}}=0$, caso contrário,

$\mathrm{S}_{0}=$ somatório dos pesos, $\mathrm{S}_{0}=\sum^{\mathrm{N}}{ }_{\mathrm{i}=1} \sum^{\mathrm{N}}{ }_{\mathrm{j}=1}$ $w_{i j}, i \neq j$. 


\section{Resultados}

\section{Aspectos Gerais}

O arquivo do Sinasc de 1994 contém 107.883 registros correspondendo ao total de nascimentos ocorridos no Município do Rio de Janeiro naquele ano, dos quais 10.364 residentes em outros municípios. A freqüência de nãopreenchimento das variáveis sexo, peso ao nascer, idade gestacional, tipo de gravidez, tipo de parto, idade da mãe e nível de instrução da mãe foi baixa, oscilando entre $0,4 \%$ (sexo) e $5,2 \%$ (idade gestacional). As variáveis Apgar no primeiro minuto e Apgar no quinto minuto apresentaram uma freqüência de não-preenchimento de $13,4 \%$ e $17,8 \%$ respectivamente. As variáveis número de filhos nascidos vivos e número de fil hos nascidos mortos não foram analisadas devido à alta freqüência de não-resposta, $44,5 \%$ e $86,0 \%$, inviabilizando os estudos sobre paridade da mãe.

Na Tabela 1 encontra-se o sumário dos indicadores selecionados para o Município e por bairros. O índice de Apgar no primeiro minuto concentra-se nos valores mais altos, com $69,6 \%$ dos nascidos vivos em boas condições; $16,7 \%$ com anóxia leve; $11,1 \%$ com anóxia moderada e 2,6\% com anóxia grave. Em relação ao peso ao nascer, $10,1 \%$ dos nascidos vivos apresentaram baixo peso; $24,7 \%$ nasceram com peso deficiente e $65,2 \%$ nasceram com peso favorável, val ores semelhantes aos encontrados em países como México e Hungria. A amplitude de variação do baixo peso por bairros foi de 5,0\% a $16,1 \%$. A proporção de recém-nascidos com peso deficiente por bairros variou entre $16,1 \%$ e $36,2 \%$, e a proporção com peso favorável ficou entre $54,8 \%$ e $73,3 \%$. A idade gestacional foi ignorada em apenas $1,2 \%$ das DNs. A freqüência de bebês prematuros foi igual a $6,7 \%$ do total de nascidos vivos, variando entre 1,8\% e $13,7 \%$ por bairros. Os partos cesáreos corresponderam a $44,7 \%$ do total, oscilando entre $28,1 \%$ e $70,7 \%$ por bairros. A faixa etária materna de vinte a 34 anos concentrou $73 \%$ dos nascidos vivos, sendo a idade mediana igual a 25 anos. A menor idade detectada foi 12 anos e a mais elevada 56 anos. Chama atenção o elevado percentual de mães adolescentes (com menos de vinte anos), correspondendo a $16,9 \%$ do total e variando entre $0,0 \%$ e $31,1 \%$ por bairros.

Observou-se um maior risco de baixo peso nos extremos etários ( Tabela 2), sendo igual a $12,8 \%$ abaixo de vinte anos, caindo para $9,1 \%$ na faixa etária de vinte a 34 anos e alcançando $13,1 \%$ para mães com 35 anos e mais. Além disso, verificou-se que as adolescentes e mães com mais de 35 anos apresentaram também maiores proporções de partos prematuros. Em relação ao tipo de parto, nota-se que entre as mães adolescentes é bem menor a proporção de cesáreas $(26,8 \%)$ do que entre as mães com idade igual ou superior a 35 anos (56,1\%).

O grau de instrução materno, na DN, está estratificado nas seguintes categorias: nenhuma instrução (2,1\%); primeiro grau incompleto $(44,2 \%)$; primeiro grau completo $(19,8 \%)$; segundo grau (21,7\%); superior (10,1\%) e ignorado $(2,1 \%)$. O baixo percentual de ignorados sugere boa qualidade da coleta. Esta variável também está relacionada com o peso ao nascer (Tabela 2), à medida que aumenta o grau de instrução, diminui a proporção de baixo peso.

Tabela 1

Indicadores selecionados: proporção no Município, média das proporções por bairros, proporção mínima e máxima e desvios-padrão, Município do Rio de J aneiro, 1994*.

\begin{tabular}{lccccc}
\hline Indicador & $\begin{array}{c}\text { Proporção global } \\
\text { no Município (\%) }\end{array}$ & $\begin{array}{l}\text { Prop. média } \\
\text { interbairros (\%) }\end{array}$ & $\begin{array}{l}\text { Proporção } \\
\text { mínima (\%) }\end{array}$ & $\begin{array}{l}\text { Proporção } \\
\text { máxima (\%) }\end{array}$ & $\begin{array}{c}\text { Desvio-Padrão } \\
\text { Prop. Apgar boma }\end{array}$ \\
Prop. peso baixob & 69,6 & 70,1 & 44,1 & 83,3 & 8,3 \\
Prop. peso deficientea & 10,1 & 10,1 & 5,0 & 16,1 & 2,8 \\
Prop. peso favorávela & 24,7 & 24,7 & 16,1 & 36,2 & 3,3 \\
Prop. prematurosc & 65,2 & 65,3 & 54,8 & 73,3 & 3,9 \\
Prop. cesáreasa & 6,7 & 6,7 & 1,8 & 13,7 & 2,0 \\
Prop. adolescentesa & 44,7 & 46,0 & 28,1 & 70,7 & 9,7 \\
Prop. 2o grau/superiora & 16,9 & 16,1 & 0,0 & 31,1 & 80,6 \\
\hline
\end{tabular}

* Excluídos os bairros que não atingiram o número mínimo de nascimentos para cada indicador.

a Excluídos os bairros com menos de vinte nascimentos.

b Excluídos os bairros com menos de 69 nascimentos.

c Excluídos os bairros com menos de 72 nascimentos. 
Também observou-se relação direta entre nível de instrução e parto cesáreo, chegando a 75,8\% entre mães com nível superior.

\section{Análise espacial}

Os boxplots dos indicadores relativos ao recém-nascido por bairros revelam que as proporções de peso baixo, peso deficiente, peso favorável e prematuros apresentam pequena amplitude de variação, com valores muito próximos, apesar de alguns valores marginais (outliers). Em oposição, as proporções de Apgar bom, mães com escolaridade acima de segundo grau e cesáreas apresentam grande amplitude de variação, ao passo que a proporção de adolescentes apresenta valores bem mais concentrados em torno da mediana (Figuras 1 e 2).

A distribuição espacial da proporção de nascidos vivos com Apgar bom no primei ro minuto por bairros é apresentada na Figura 3. Nota-se concentração dos valores mais altos nos bairros da Zona Sul, Centro, trechos da Zona Norte e da Il ha do Governador, parecendo haver uma tendência de diminuição dos valores em direção à Zona Oeste. Devido ao percentual relativamente alto de não-resposta da variável Apgar no primeiro minuto $(13,4 \%)$, foi mapeada a proporção de registros com esta variável em branco por bairros (Figura 4), revelando que, dos 153 bairros estudados, esta proporção esteve abaixo de $10 \%$ em 124 deles, o que pode ser consi derado como razoável. Houve 17 bairros com percentual entre $10 \%$ e $20 \%$, considerado elevado. Em 13 bairros este percentual ficou entre $20 \%$ e $50 \%$, muito elevado, localizados principalmente na Zona Oeste da cidade, e dois bairros atingiram $50 \%$ de registros em branco (Santa Cruz e Sepetiba). Este aspecto provavel mente está relacionado com a qualidade da assistência ao parto nos hospitais onde a maioria desta população é atendida. Os resultados para estes bairros devem ser analisados com cautela.

Na Figura 5, observamos a distribuição espacial da proporção de baixo peso por bairros. Esta se assemelha a um mosaico, não sendo possível identificar visualmente qualquer tipo de padrão, em contraste com o mapa anterior. As proporções de peso deficiente e favorável apresentaram comportamento semelhante, o que poderia ser esperado ao se visualizarem os boxplots destes indicadores. A baixa amplitude de variação traduz-se, nos mapas de padrão, em classes muito próximas, com pequena capacidade de diferenciação. Todos os indicadores de peso ao nascer construídos excluindo-se os bebês prematuros apresentaram distribui-
Tabela 2

Proporções de baixo peso, prematuros e cesáreas segundo idade e escolaridade da mãe, Município do Rio de J aneiro, 1994.

\begin{tabular}{lrcc}
\hline & Prop. baixo peso & Prop. prematuros & Prop. cesáreas \\
\hline Idade da mãe & & & \\
Menos de 20 & 12,8 & 8,1 & 26,8 \\
20 a 34 & 9,1 & 6,2 & 46,8 \\
35 e mais & 13,1 & 8,5 & 56,1 \\
& & & \\
Instrução da mãe & & & 25,2 \\
N enhuma & 12,1 & 6,8 & 29,9 \\
1o grau incompleto & 11,5 & 7,1 & 44,8 \\
1ㅇ grau completo & 10,1 & 6,8 & 62,8 \\
2o grau & 8,1 & 5,9 & 75,8 \\
Superior & 6,8 & 5,6 & \\
\hline
\end{tabular}

Figura 1

Boxplots das proporções de baixo peso, peso deficiente, peso favorável, Apgar bom e prematuridade por bairros, Município do Rio de J aneiro, 1994.

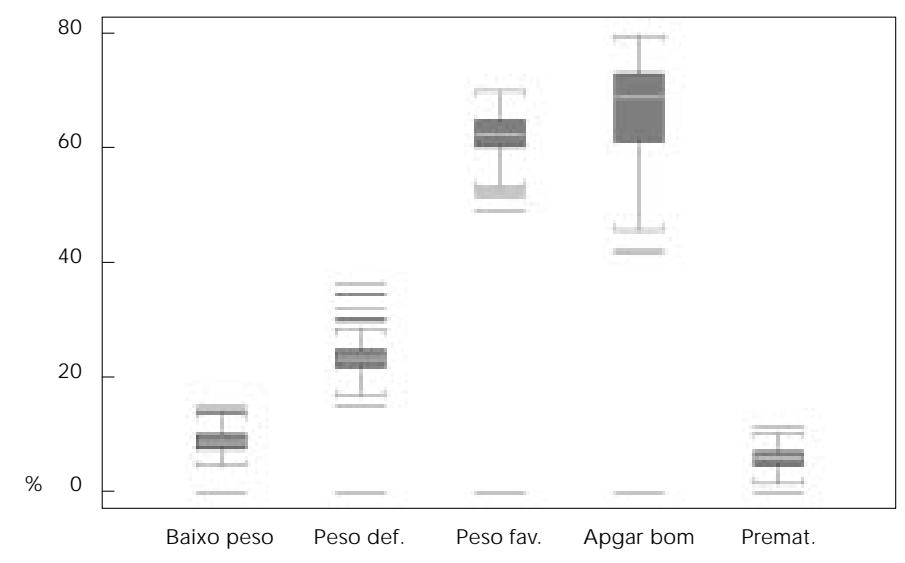

ção espacial aleatória. Este fato aponta para a superioridade de um índice composto, como o Apgar, em relação a um indicador simples, como o baixo peso, na caracterização de áreas de risco nesta escala de análise.

Na Figura 6, encontra-se a distribuição espacial da prematuridade. Praticamente toda a Zona Oeste classificou-se no quintil inferior, mostrando claramente um cluster de baixos valores de prematuridade nesta área, enquanto o restante da cidade apresenta uma situação de mosaico. Este é um achado inesperado, uma vez que se trata justamente da região mais carente do Município. Deve ser acrescentado 
Figura 2

Boxplots das proporções de adolescentes, cesáreas e mães com escolaridade acima de segundo grau por bairros, Município do Rio de J aneiro, 1994.

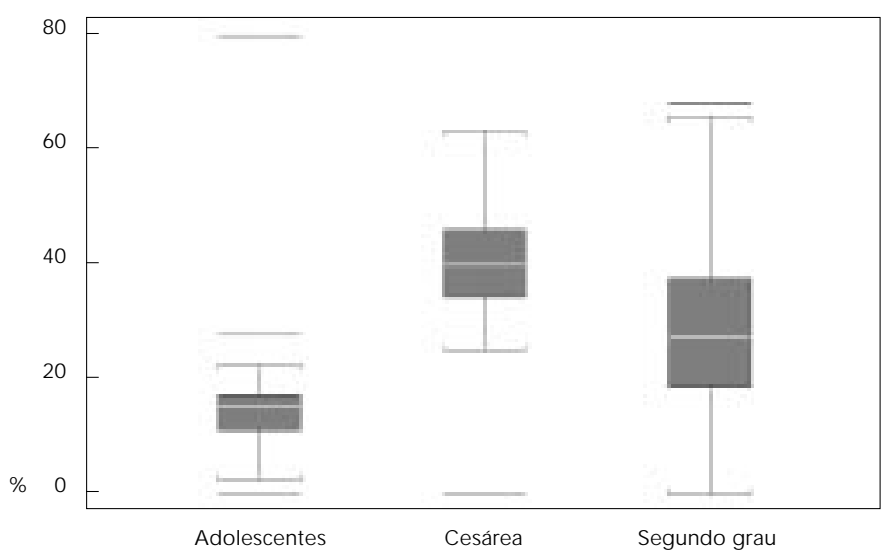

que, em nível individual, a prematuridade não apresentou correlação com nenhum outro indicador, exceto baixo peso.

A distribuição espacial da proporção de cesáreas (Figura 7) mostrou concentração dos valores mais elevados nos bairros das Zonas Sul e Norte e valores mais baixos na Zona Oeste, sugerindo que este indicador apresenta relação direta com o nível sócio-econômico da área, fato apontado por diversos autores (Goldman et al., 1993; Bertollini et al., 1992; MMWR, 1993).

A Figura 8 (proporção de mães adolescentes por bairros) apresenta padrão inverso ao do mapa anterior, com valores mais baixos de mães adolescentes naquelas áreas onde a proporção de cesáreas é mais el evada, indicando que os bairros com menos infra-estrutura são aqueles com maiores proporções de mães adolescentes e menores taxas de cesáreas. A distribuição espacial da proporção de mães com escolaridade acima de segundo grau (Figura 9) é semelhante à de Apgar bom, cesáreas e adolescentes.

\section{A uto correlação}

$\mathrm{Na}$ Tabela 3, encontram-se os resultados do teste I de Moran para autocorrelação espacial das variáveis estudadas. Conforme o esperado pela inspeção visual dos mapas, a proporção de Apgar bom apresenta correlação alta $(0,55)$. Os indicadores referentes à escolaridade e à idade da mãe, embora ainda significativos, encontram-se em patamar inferior. A proporção de cesáreas e de recém-natos prematuros apresentaram autocorrelação pequena $(0,20$ e 0,18 respectivamente). Observa-se a diferença entre estes nos mapas, onde o padrão espacial da prematuridade pode ser descrito como aleatório, exceto pela concentração de baixos valores na Zona Oeste. Já o mapa das cesáreas deve seu índice de autocorrelação a uma estrutura espacial distribuída em toda a área. O indicador de baixo peso não tem qualquer autocorrelação espacial, confirmando que nesta escala a variável não é espacialmente condicionada.

\section{Discussão}

A taxa de cesáreas, tanto para o Município, como por bairros, é bastante elevada, sendo o menor valor por bairro $(28,1 \%)$ praticamente o dobro do valor de $15 \%$ recomendado pela OMS, com base em indicações clínicas (MMWR, 1993). Quando relacionada à escolaridade materna, observa-se a clara relação entre nível sócio-econômico e tipo de parto. A cesárea é um recurso que deve ser utilizado quando o desenrolar normal do parto implica algum tipo de risco para a mãe, o feto ou ambos. Como todo procedimento cirúrgico, a cesárea não é isenta de riscos, estando associada a uma maior morbi-mortalidade materna e infantil. A morbidade materna por complicações cirúrgicas inclui laceração acidental, hemorragia, infecção puerperal, embolia pulmonar, íleo paralítico e reações indesejáveis à anestesia. Para o recémnascido, estão relatadas maior freqüência de síndrome de angústia respiratória e prematuridade iatrogênica (Miller, 1988). Além disso, o parto cesáreo implica um maior tempo de recuperação para a puérpera, interferindo no estabelecimento da relação mãe-filho no pósparto e no início precoce e bem-sucedido da amamentação. Também compromete a futura história reprodutiva, pela alta freqüência de cesáreas de repetição e realização concomitante de laqueadura tubárea.

Muitos estudos têm demonstrado associação positiva entre taxas de cesáreas e padrão sócio-econômico (Rattner, 1996, Campos 1997, Chacham \& Perpétuo, 1996). Estes achados apontam para a incoerência de se encontrar uma maior freqüência de partos operatórios justamente na população com melhor padrão sócio-econômico, que teoricamente apresentaria um menor risco gestacional e necessitaria menos deste tipo de intervenção. Entretanto, os valores entre a população de menor padrão sócio-econômico não podem ser considerados ideais, uma vez que são apenas mais baixos. Estes trabalhos indicam que a cesárea parece 


\section{Figura 3}

Proporção de nascidos vivos com Apgar bom por bairros, Município do Rio de J aneiro, 1994.

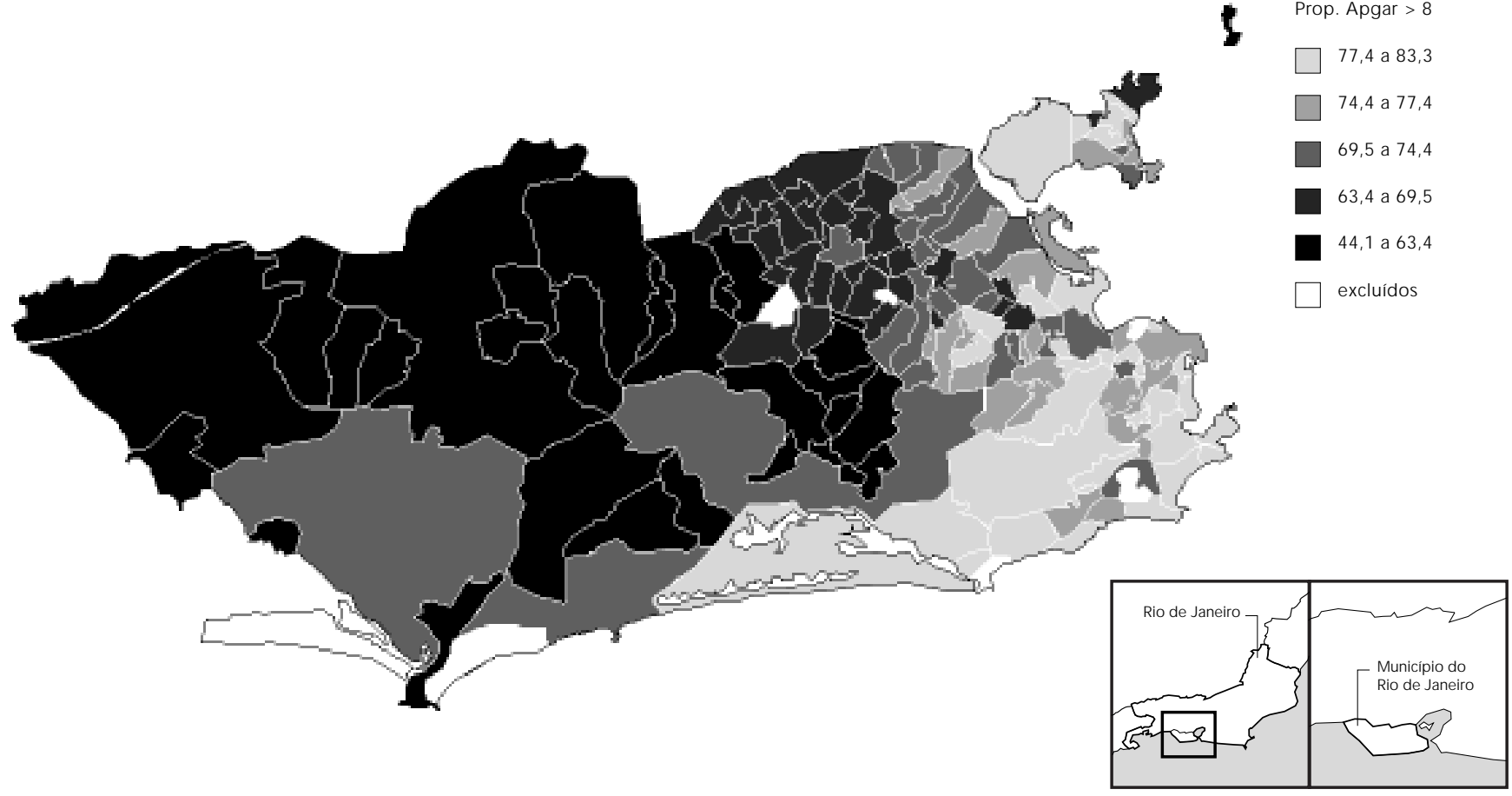

Fonte: SINASC

\section{Figura 4}

Proporção de não-resposta do Apgar no primeiro minuto por bairros, Município do Rio de J aneiro, 1994.

1. Prop. não-resposta,

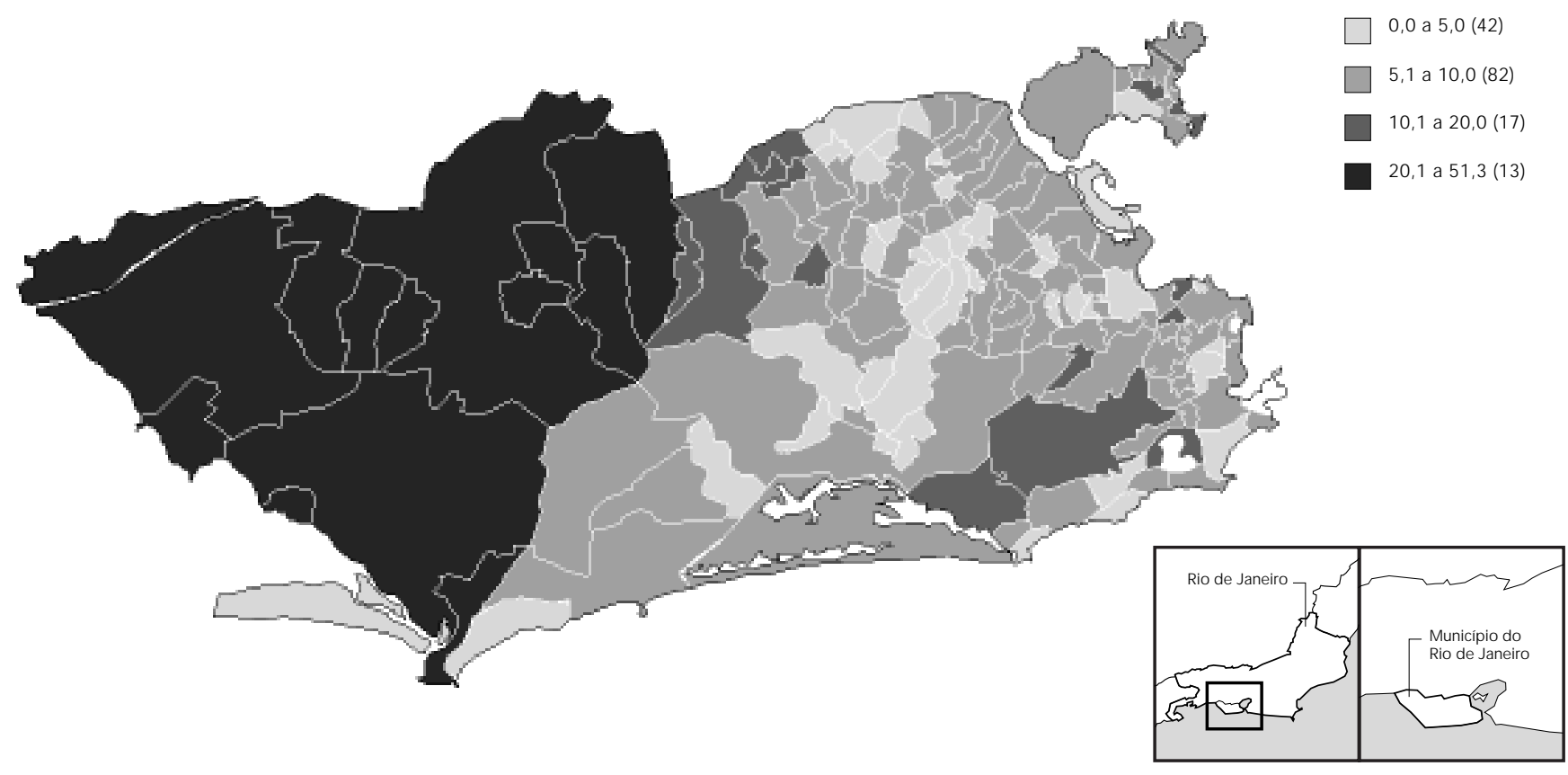




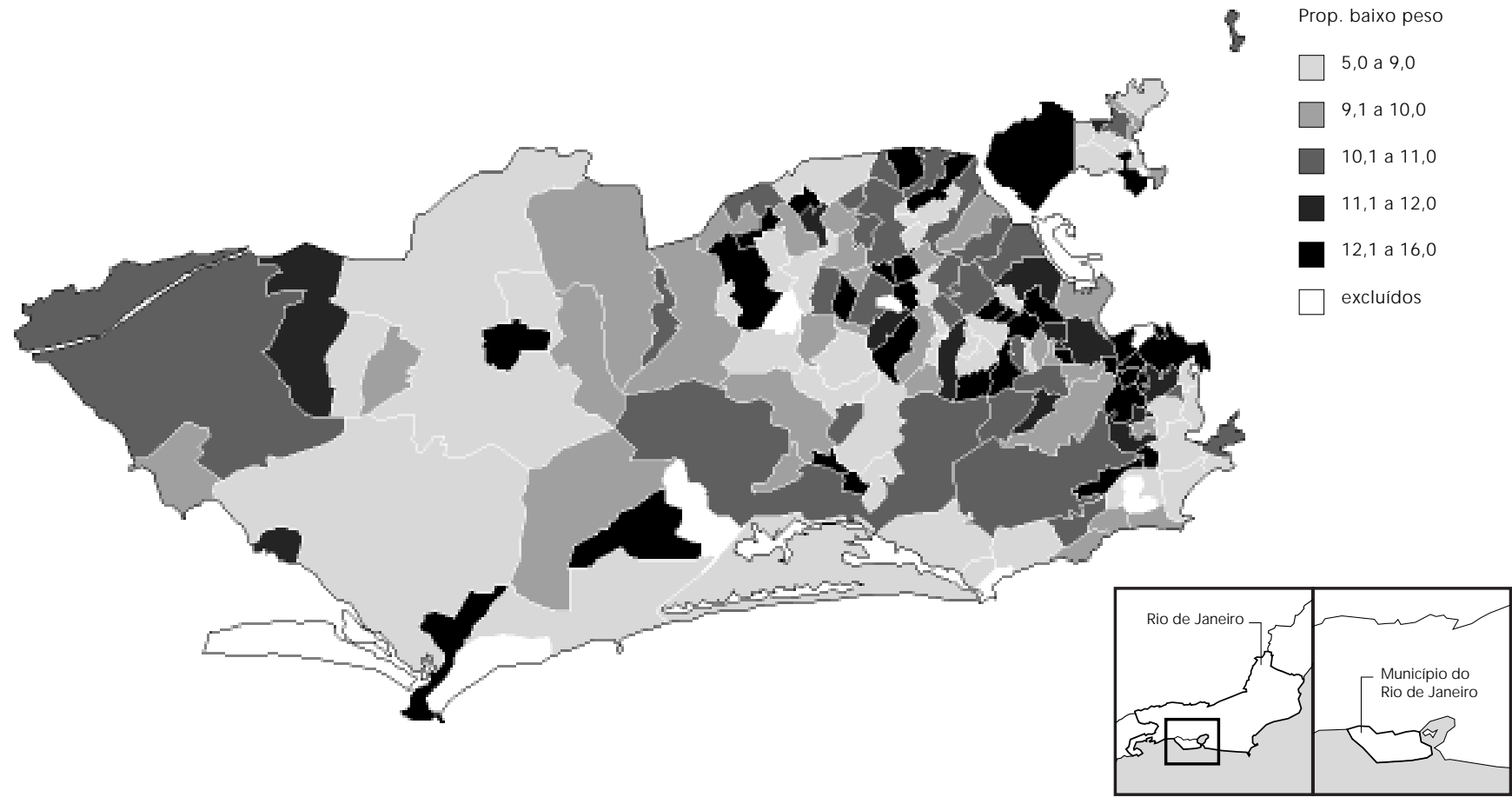

Fonte: SINASC

Figura 6

Proporção de prematuros por bairros, Município do Rio de J aneiro, 1994.

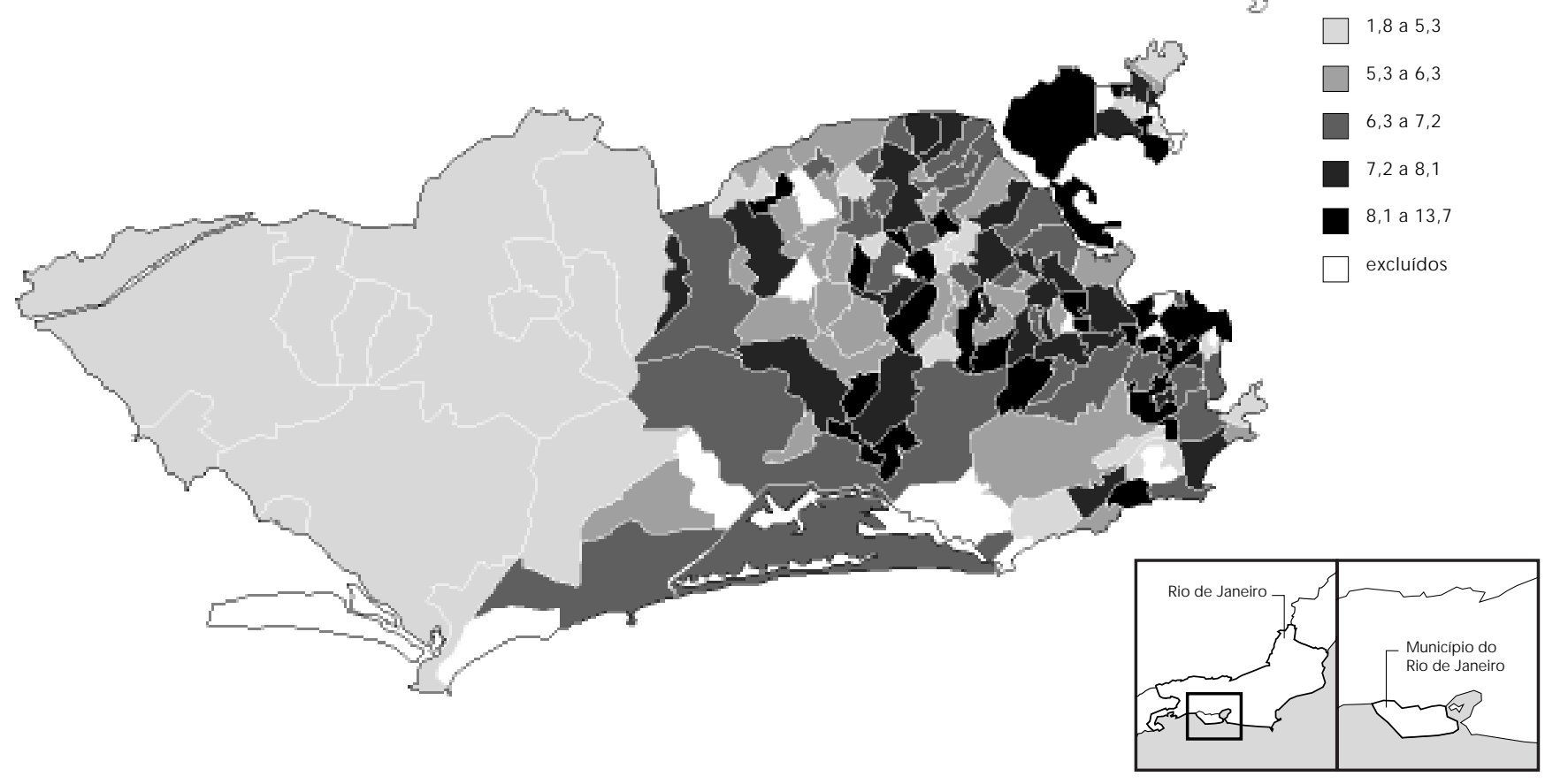




\section{Figura 7}

Proporção de cesáreas por bairros, Município do Rio de J aneiro, 1994.

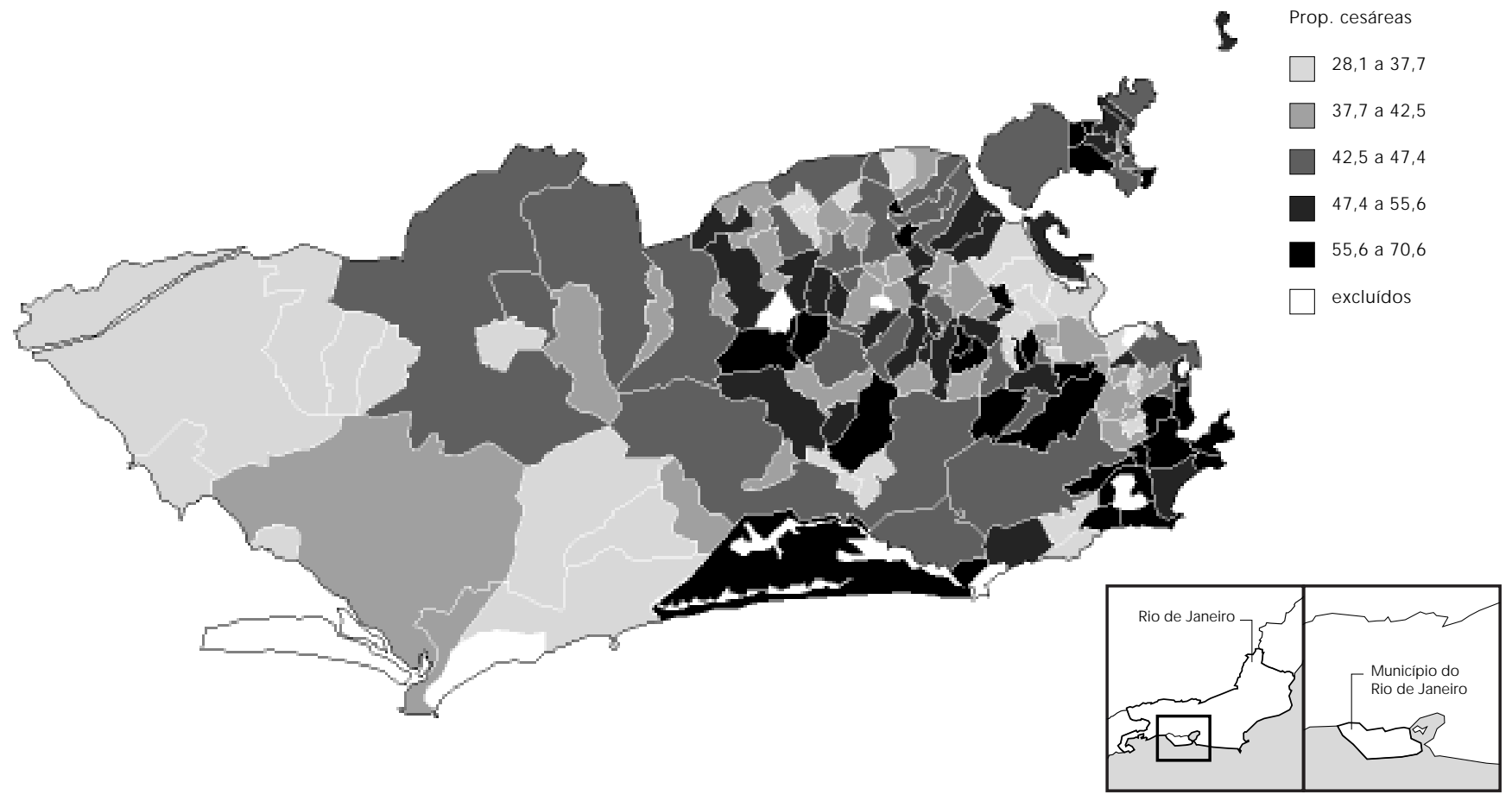

Fonte: SINASC

\section{Figura 8}

Proporção de mães adolescentes por bairros, Município do Rio de J aneiro, 1994.

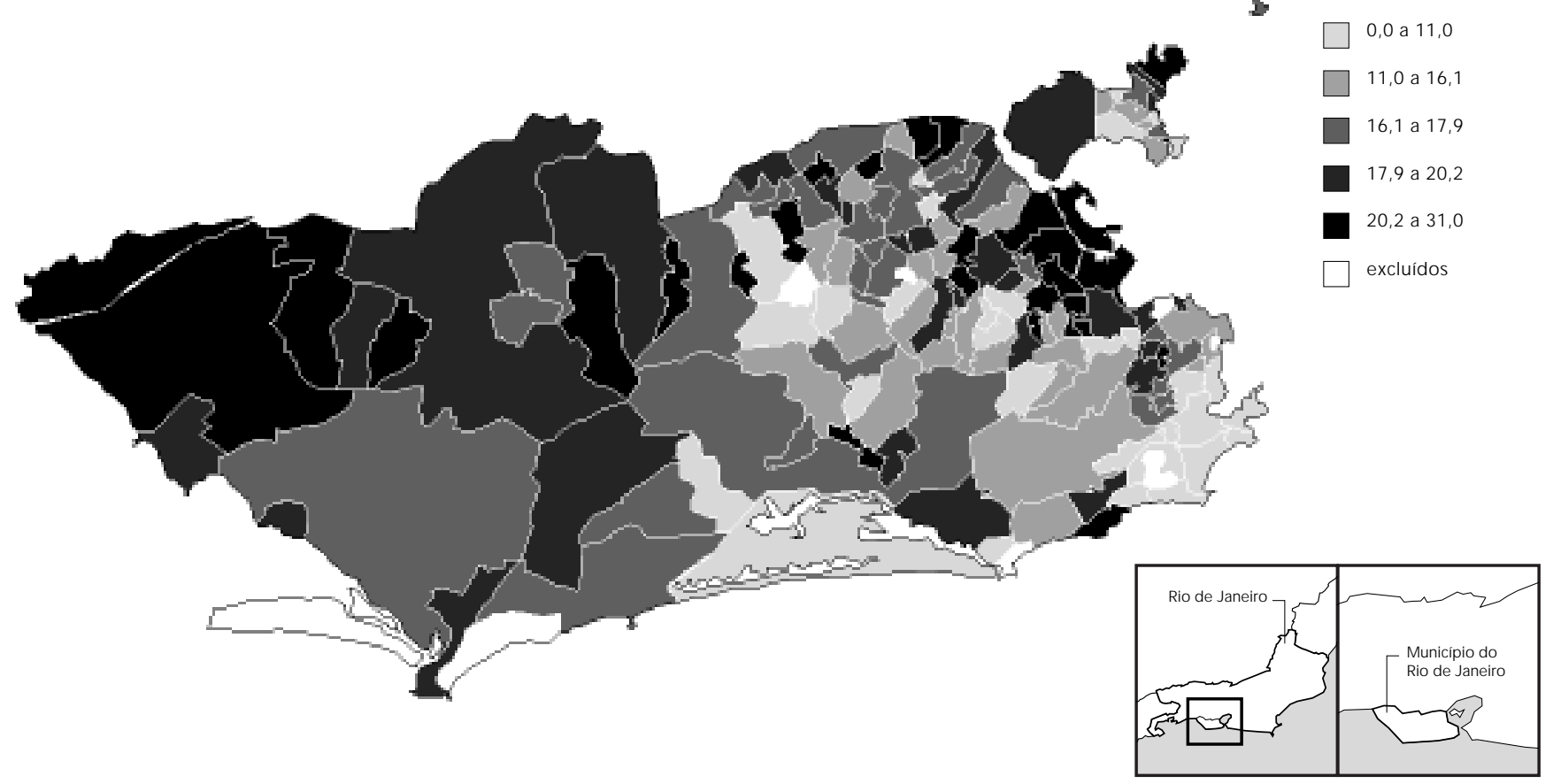




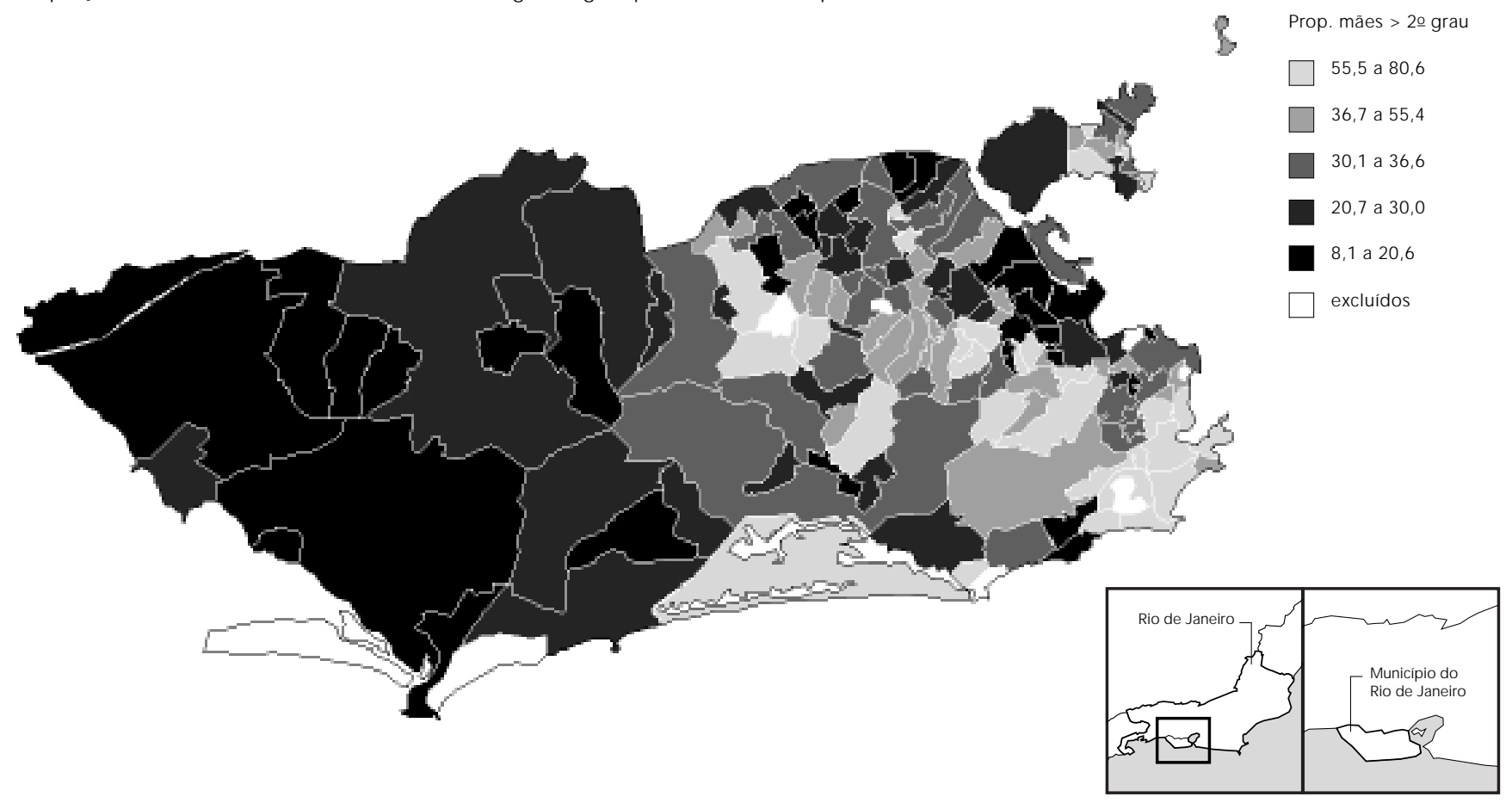

ter se tornado um bem de consumo, dependente do poder aquisitivo da população.

O baixo peso apresentou padrão espacial aleatório nesta escala de análise; a prematuridade apresentou um padrão homogêneo apenas na Zona Oeste, com valores baixos, e padrão de mosaico no restante do Município, com baixa autocorrelação espacial. Dado que baixo peso e prematuridade são variáveis correlacionadas, a diferença no padrão de distribuição espacial leva a questionar a qualidade de resposta desta variável. O total de não-resposta da idade gestacional foi apenas 5,2\%; as principais maternidades que atendem à Zona Oeste apresentam valores em torno da média municipal para o indicador, exceto por uma delas, onde apenas 1,7\% dos recém-natos tem idade gestacional inferior a 37 semanas (Campos, 1997). Como baixos valores de prematuridade é um achado pouco consistente com as demais informações, particularmente com a distribuição do baixo peso e da escolaridade da mãe, é possível que seja conseqüência da qualidade do registro dos dados nos hospitais onde esta população é atendida. Deve ser lembrado que qualidade da informação é um indicador indireto da qualidade da assistência.
Quanto à aleatoriedade espacial na distribuição do baixo peso ao nascer, cabe lembrar que os bairros são unidades heterogêneas quanto ao nível sócio-econômico da população, relacionado aos fatores determinantes da ocorrência de baixo peso e prematuridade. É possível que, alterando-se a escala, possa-se observar um padrão espacial não aleatório do peso ao nascer, conforme encontrado por outros autores (Victora et al, 1994). Esta mesma lógica, entretanto, implicaria perfil semelhante na distribuição do índice de Apgar, ao invés do padrão geográfico encontrado. Como explicar, então, a autocorrelação espacial encontrada para a proporção de recém nascidos com Apgar bom?

O baixo peso está ligado basicamente a problemas biológicos, nutricionais e prematuridade, que, por sua vez, está associada a intercorrências maternas, crônicas ou agudas, durante a gestação. Em nível individual, é inquestionável que a gestante com menor escolaridade e com uma maior freqüência de patologias durante a gestação apresente um maior risco de parto prematuro, recém-nascido de baixo peso e conseqüente maior risco de morbi-mortalidade neonatal. Entretanto, a análise de gru- 
pos populacionais, compostos pelas gestantes residentes em bairros cariocas, com seu gradiente quanto ao nível sócio-econômico, sugere que os determinantes de prematuridade e baixo peso não têm ocorrência significativamente diferente entre os bairros. Para que se pudessem detectar desigual dades no comportamento destes grupos, a prevalência de gestantes com patologias ou com inadequada assistência pré-natal deveria também ser diferente entre os bairros, o que, aparentemente, não ocorre. É o caso, por exemplo, da desnutrição materna, inexistente no Rio de Janeiro em níveis importantes e que apresentem diferencial interbairros mensurável. Outros eventos que levam ao baixo peso são raros: toxemia gravídica, anomalias congênitas, entre outros, e não permitem diferenciar grupos.

O Apgar, por outro lado, é um indicador mais diretamente relacionado à qualidade da assistência no momento do parto. Apesar da influência das condições prévias do recémnascido durante o período intra-uterino, que determinam a vitalidade no momento do nascimento, uma má assistência, onde ocorra sofrimento fetal, pode implicar um recém-nato com Apgar abaixo de oito, mesmo em gestações a termo com peso adequado.

A capacidade de um indicador em detectar populações de risco varia conforme o local e a escala de análise. Em situações onde o diferencial sócio-econômico reflete-se diretamente no aporte nutricional da gestante, como, por exemplo, na Índia, a incidência de baixo peso ao nascer terá grande importância na identificação de grupos de risco. Em locais como o Rio de Janeiro, onde as condições de saúde da gestante não são significativamente desiguais entre os bairros, a detecção dos grupos de maior risco será relacionada a outros indicadores.

Corrobora esta análise o trabal ho de Campos (1997), mostrando o fluxo do local de residência para o local de ocorrência do óbito de crianças que faleceram com menos de 28 dias, que apresenta clara associação com o padrão sócio-econômico dos bairros - a Zona Oeste 'exportando' recém-nascidos cujos óbitos vão ocorrer em hospitais localizados no Centro e na Zona Sul do Município. A mortalidade neonatal (cujos principais determinantes são a prematuridade e o baixo peso) no Rio de Janeiro também apresentou padrão espacial aleatório, seja segundo municípios do Estado (Leal, 1996), seja segundo bairros do Município do Rio de Janeiro, indicando que "a mudança no perfil da mortalidadeinfantil ea possibilidade de intervenção desl oca-se cada vez mais para a
Tabela 3

Teste I de Moran para os indicadores selecionados e significância segundo suposições de normalidade e aleatoriedade. Município do Rio de J aneiro, 1994.

\begin{tabular}{lccc}
\hline Indicador & Moran I & Signif. N & Signif. R \\
\hline Prop. Apgar bom & 0,5521 & 0,0000 & 0,0000 \\
Prop. baixo peso & 0,0776 & 0,1162 & 0,1161 \\
Prop. prematuros & 0,1889 & 0,0003 & 0,0003 \\
Prop. cesáreas & 0,2046 & 0,0000 & 0,0000 \\
Prop. adolescentes & 0,2618 & 0,0053 & 0,0007 \\
Prop acima de 2o grau & 0,2914 & 0,0000 & 0,0000 \\
\hline
\end{tabular}

esfera dos serviços de saúde, especialmente os médico-assistenciais" (Campos, 1997). Assim, o grande diferencial sócio-econômico entre as gestantes reflete-se, hoje, na assistência de boa qualidade ao parto, e o índice de Apgar assume importância crescente como indicador de risco.

O baixo peso ao nascer torna-se assim, pelas suas características epidemiológicas, menos um indicador de risco agregado, e mais um evento-sentinela de uma ocorrência potencialmente evitável: o óbito infantil. Outros estudos permitiriam aprofundar esta análise, seja diminuindo a escala e investigando a ocorrência de baixo peso em macrorregiões com grandes contrastes, por exemplo municípios da Região Sudeste, seja aumentando a escala e investigando microáreas (setores censitários) de uma grande metrópole. Neste caso poder-se-iam detectar grupos particularmente vulneráveis, nos quais ainda é grande número de crianças com baixo peso ao nascer, como é o caso de famílias de rua ou de áreas extremamente carentes na expansão das favelas.

\section{Conclusões}

Os indicadores que mel hor caracterizaram os bairros do Rio de Janeiro foram as proporções de: nascidos vivos com Apgar bom no primeiro minuto, mães com escolaridade acima do segundo grau e mães adolescentes. O baixo peso, considerado a principal variável preditiva de risco para a vigilância do recém-nascido, apresentou distribuição espacial aleatória. O aparente contraste entre a distribuição al eatória do baixo peso (reconhecidamente ligado às condições de vida da família) e a coincidência de baixos valores de Apgar no primeiro minuto com os indicadores de pobreza deve ser atribuído à qualidade da assistência ao parto prestada nas áreas carentes do Município. A precá- 
ria qualidade destes serviços deve ser a verdadeira causa da melhor adequação do Apgar aos indicadores de condições de vida, apesar de não o ser na literatura mundial. Ou seja, se em termos individuais o baixo peso é adequado enquanto preditor de risco infantil, na identificação de áreas ou grupos populacionais, particularmente quando o grande diferencial é o acesso ao serviço de saúde, o Apgar, ainda pouco valorizado, parece ser mais útil. O registro deste indicador deve ser estimulado nos hospitais, uma vez que consiste em uma medida simples, que não necessita da presença de um neonatologista na sala de parto, podendo ser realizada por pessoal de enfermagem treinado.

\section{Referências}

ABREU, M. A., 1987. A Evolução Urbana do Rio de Janeiro. Rio de Janeiro: I planrio/Zahar.

BERKOWITZ, G. S. \& PAPIERNIK, E., 1993. Epidemiology of preterm birth. Epidemiologic Reviews 15: 414-442.

BERTOLLINI, R.; DILALLO, D.; SPADEA, T. \& PERUC$\mathrm{Cl}, \mathrm{C} ., 1992$. Cesarean section rates in Italy by hospital payment mode: an analysis based on birth certificates. American Journal of Public Health, 82:257-261.

CAM POS, T. P., 1997. Perfil de Nascimentos é Óbitos Infantis: A Busca da Assistência no Município do Rio de Janeiro. Dissertação de Mestrado, Rio de Janeiro: Escola Nacional de Saúde Pública, Fundação Oswaldo Cruz.

CHACHAM, A. S. \& PERPÉTUO, I. H. O., 1996. Determinantes sócio-econômicos da incidência de partos cirúrgicos em Belo Horizonte. Anais do X Encontro Nacional deEstudos Populacionais, vol. 4:2.587-2.610.

CLIFF, A. D. \& ORD, J. D., 1981. Spatial Processes, Model and Application. London: Pion.

FAÚNDES, A. \& CECATTI, J. G., 1991. A operação cesárea no Brasil. Incidência, tendências, causas, conseqüências e propostas de ação. Cadernos de SaúdePública, 7:150-173.
O excesso de cesáreas, principalmente nos bairros da Zona Sul da cidade, indicam uma distorção da prática obstétrica. É necessário investigar as razões de tal distorção e implementar ações que estimulem a prática mais freqüente de partos espontâneos.

Esta metodologia mostrou-se útil para um melhor conhecimento sobre o perfil de nascimentos no Município do Rio de Janeiro, sendo a análise espacial por bairros interessante por revelar importantes diferenciais em relação às variáveis estudadas, podendo contribuir para o planejamento de ações voltadas para a saúde materno-infantil que sejam dirigidas para áreas com características específicas.
FERRAZ, E. M.; GRAY, R. H. \& CUNHA, T. M., 1990. Determinants of preterm delivery and intrauterine growth retardation in North-East Brazil. International Journal of Epidemiology, 19:101-107.

GOLDMAN, G.; PINEAULT, R.; POTVIN, L.; BLAIS, R. \& BILODEAU, H., 1993. Factors influencing the practice of vaginal birth after cesarean section. American Journal of Public Health, 83:1.1041.108.

GRAY, R. H.; FERRAZ, E. M.; AM ORIM, M. S. \& MELO, L. F., 1991. Levels and determinants of early neonatal mortality in Natal, Northeastern Brazil: results of a surveillance and case-control study. International Journal of Epidemiology, 20:467473.

HAYES, M. V., 1992. On the epistemology of risk: Ianguage, logic and social science. Social Scienceand Medicine, 35:401-407.

KALLAN, J. E., 1993. Race, intervening variables, and two components of low birth weight. Demography, 30:489-506.

LEAL, M. C., 1996. Evolução da Mortalidade Infantil no Estado do Rio de Janeiro na Década de 80: Componente Neonatal. Tese de Doutorado, Rio de Janeiro: Escola Nacional de Saúde Pública, Fundação Oswaldo Cruz. 
MAVALANKAR, D. V.; GRAY, R. H. \& TRIVEDI, C. R., 1992. Risk factors for preterm and term low birthweight in Ahmedabad, India. International Journal of Epidemiology, 21:263-272.

MELLO JORGE, M. H. P.; GOTLIEB, S. L. D.; SOBOLL, M. L. M. S.; BALDIJÃO, M. F. A. \& LATORRE, M. R. D. O., 1992. O Sistema de Informações sobre Nascidos Vivos - SINASC. São Paulo: Centro Brasileiro para a Classificação de Doenças/ OPAS-OMS, Núcleo de Estudos em População e Saúde (NEPS/ USP).

MELLO JORGE, M. H. P.; GOTLIEB, S. L. D.; SOBOLL, M. L. M. S.; BALDIJÃO, M. F. A. \& LATORRE, M. R. D. O., 1993. Avaliação do Sistema de Informação sobre Nascidos Vivos e o uso de seus dados em epidemiologia e estatísticas de saúde. Revista de SaúdePública, 27(supl.):22-37.

MILLER, J. M., 1988. Maternal and neonatal morbidity and mortality in cesarean section. Obstetrics and Gynecology Clinics of North America, 15:629638.

MMWR, 1993. Rates of cesarean delivery - United States, 1991. Morbidity and Mortality Weekly Report, 42:285-289.

PNAD (Pesquisa Nacional por Amostra de Domicílio), 1996. Pesquisa Nacional por Amostra de Domicílio. Rio de Janeiro: Fundação Instituto Brasileiro de Geografia e Estatística (FIBGE).

PORRECO, R. P. \& THORP, J. A., 1996. The cesarean birth epidemic: trends, causes and solutions. American Journal of Obstetrics and Gynecology, 175:369-374.
PUFFER, R. R., 1987. Patterns of Birthweights. Scientific Publication, 504. Washington: Pan American Health Organization.

RATTNER, D., 1996. Sobre a hipótese de estabilização das taxas de cesárea do Estado de São Paulo. Revista de Saúde Pública, 30:19-33.

SANJOSE, S. \& ROMAN, E., 1991. Low birthweight, preterm, and small for gestational age babies in Scotland, 1981-1984. Journal of Epidemiology and Community Health, 45:207-210.

THIERY, M. \& DERON, R., 1986. Review of evaluation studies on cesarean section. Part I - Trends in cesarean section and perinatal mortality. In: Perinatal Care Delivery Sistems: Description and Evaluation in European Community Countries (M. Kaminsky et al., eds.), pp. 93-113, Oxford: Oxford Community Press.

VICTORA, C. G.; GRASSI, P. R. \& SCHMIDT, A. M, 1994. Situação de saúde da criança em área da região Sul do Brasil, 1980-1992: tendências temporais e distribuição espacial. Revista de Saúde Pública, 28:423-432.

WILCOX, A. J. \& SKJFOERVEN, R., 1992. Birth weight and perinatal mortality: the effect of gestational age. American Journal of Public Health, 82:378382. 